\title{
Natural Resistance Against Brucellosis: A Review
}

\author{
L. Garry Adams ${ }^{*}, 1$ and Christopher J. Schutta ${ }^{2}$ \\ ${ }^{I}$ Department of Veterinary Pathobiology, College of Veterinary Medicine, Texas A\&M University, College Station, \\ Texas, TX 77843-4467, USA \\ ${ }^{2}$ Department of Homeland Security, Science \& Technology Directorate, Targeted Advanced Development Unit, Plum \\ Island Animal Disease Center, P.O. Box 848, Greenport, New York, NY 11944, USA
}

\begin{abstract}
Natural resistance against brucellosis was reviewed from historical and biological perspectives with regard to animals, humans and Brucella spp. Unfortunately, brucellosis continues to be a serious worldwide bacterial zoonosis of major significance to animal and human populations. Host genetic, innate and adaptive immune factors significantly influence the outcome of brucellosis as does the enabling strategies of intracellular Brucella to evade host factors resulting in a delicate co-evolutionary balance for long term survival for both host and pathogen. Natural (innate) resistance mechanisms include the complex of host cell surface receptors for Brucella pathogen-associated molecular patterns, TollIL-1 receptor mediated pathways, factors mediating effective macrophage and dendritic cell maturation and activation, carbohydrate binding proteins, antimicrobial peptides, and inflammatory cytokines orchestrated and regulated by the host genome. Heritability of natural resistance has long been recognized as a complex multigenic trait, however new tools for understanding the genetic basis for innate resistance are now providing a deeper knowledge to identify genes and polymorphisms associated with resistance or susceptibility. Polymorphisms of the 3'UTR of the candidate solute carrier gene, SLC11A1, have been investigated extensively in numerous host species yielding contradictory variable degrees of association with natural resistance to brucellosis in ruminants, and indicating the need for international standardized phenotyping protocols. By coupling new genetic tools with rigorously controlled phenotyping protocols, it is anticipated that applying genetic selection as an additional approach to controlling infectious diseases, such as brucellosis, in domestic animals will become increasingly feasible in future.
\end{abstract}

Keywords: Natural resistance, genetic resistance, ruminants, Brucellosis, NRAMP1, SLC11A1.

\section{INTRODUCTION}

\section{The Disease}

Brucellosis is a major worldwide bacterial zoonosis. It has remained a disease of global importance since its discovery by Bruce in $1888[1,2]$. The causative agent of brucellosis is a group of gram-negative facultative intracellular bacteria belonging to the genus Brucella. The members of this genus are subdivided into seven species categorized by antigenic variation and primary preferred host: Brucella melitensis (sheep and goats), B. abortus (cattle), B. canis (dogs), B. suis (hogs), B. ovis (sheep), B. neotomae (rats) and $B$. maris (marine mammals) [3]. Given that genetic factors and innate immune receptors significantly influence the outcome of infectious diseases, the primary host preference exhibited by the individual Brucella species reflects an extremely complicated battle of two genomes and the co-evolutionary balance between the pathogen's genome, which has evolved strategies enabling survival within the preferred host $[4,5]$, and the primary host genome, which has evolved strategies of innate and adaptive immune responses which suppress pathogen expansion usually through redundant host biological systems [6-10].

*Address correspondence to this author at the Department of Veterinary Pathobiology, College of Veterinary Medicine, Texas A\&M University, College Station, Texas, TX 77843-4467, USA; Tel: 979-845-9816; Fax: 979-862-1088; E-mail: gadams@cvm.tamu.edu
Why then is genetic resistance not being used more in modern livestock industries since it is not especially due to the lack of evidence of genetic control of disease resistance? Perhaps it is because regulatory officials, owners, producers and other industry managers do not recognize the potential of genetic resistance, not necessarily as the tool to replace traditional methods of disease control, but to add another approach to reduce the impact of bacterial pathogens on animal health and to play a role in system-based approaches, such as the pre-harvest pathogen reduction program. Significant losses caused by bacterial diseases continue to curtail livestock industries despite traditional control measures. These problems and concerns suggest that other measures to control infectious disease should be sought to enhance animal health management programs. Newer strategies to increase the overall level of resistance at herd and population levels by using selective breeding programs to enhance natural resistance would be expected to contribute significantly in this regard. The ability to effectively and economically apply genetic selection to the problem of controlling infectious diseases in domestic animals will become increasingly feasible in the future. Investigators in several branches of science are bringing us even closer to this aim by providing basic information on the genetic constitution of domestic animals, specific genes controlling mechanisms and standardized phenotyping protocols applied in innate and adaptive resistance to bacterial diseases. Utilization of such basic information to enhance herd health would be a significant adjunct to current 
and future bacterial disease control modalities for diseases of domestic animals such as brucellosis within the next decades.

Worldwide brucellosis continues to be a major economic and public health concern. Geographical regions listed as high risk includes the Mediterranean Basin, South and Central America, Eastern Europe, Asia, Africa, the Caribbean, and the Middle East [11]. Of even greater concern is the fact that brucellosis is considered a reemerging problem for countries such as Israel, Kuwait, Saudi Arabia, Brazil and Colombia [12]. World Health Organization data documents approximately 500,000 human cases of brucellosis per year [11]. The absence of a human vaccine means that along with pasteurization and proper sanitation, the prevention of human infection requires control of the disease in the animal hosts. The epidemic potential of Brucella species, the efficiency of aerosol infection, and the lack of an efficient human vaccine also make this airborne pathogen a potential agent of bioterrorism [3]. In fact, successful attempts at weaponizing $B$. suis occurred as early as 1954 [13]. In 1999, a suspected case of human brucellosis with an atypical clinical presentation prompted an investigation of possible bioterrorism in New Hampshire and Massachusetts [14], thus with very few exceptions, virtually all members of the genus Brucella are now classified as biosafety level 3 pathogens and have been placed on the Select Agent list of Bioterrorism as part of the 2001 U.S. Patriot Act [15].

\section{The Natural History of Brucellosis}

In most hosts, Brucella exposure occurs orally, aerosol or transplacentally, except in dogs, sheep, and swine in which brucellae are also sexually transmitted. Like other intracellular pathogens, brucellae require four major steps for successful infection: adherence, invasion, establishment, and dissemination [16]. The exact mechanism of bacterial entry into the host cell is not known [3]; however, evidence supporting a role for pattern recognition receptors (PRRs), including leukocyte function-associated antigen 1 (LFA-1), complement receptor 3 (CR3), alpha-5 beta-1 and alpha-V beta-3 integrins, mannose-6-phosphate receptors (MPR), and Fc receptors, supports the existence for B. abortus pathogenassociated molecular patterns (PAMPS) binding macrophages mediating the binding and internalization of the pathogen [17]. More recently, Kim and Watarai et al. indentified lipid raft microdomain interactions between Brucella and class A scavenger receptors [18-21]. Additionally, Capparelli et al. found that the haplotype pair HYA/HYA at the MBL (mannose binding lectin) locus of water buffalo is associated with resistance to $B$. abortus infection and the haplotype pairs LYD/LYD with susceptibility. Inhibition of the antibacterial activity following heat treatment of the serum, addition of specific MBL inhibitors or anti-human MBL antiserum provided evidence that the antibacterial activity was related to the serum MBL [22].

The intracellular niche of infecting brucellae includes the early Brucella containing vacuoles (BCVs) and later the specialized acidified phagosome within the macrophages of the host, called brucellasomes [23-25]. Qin, Pei et al. succinctly reviewed these steps: Brucella are internalized from the host cell plasma membrane and orchestrate the biogenesis of early Brucella-containing vacuoles (BCVs); BCVs acidify but fail to accumulate mannose 6-phosphate receptors and cathepsin D as markers for late endosomes and lysosomes, respectively; rather than maturing, BCVs fuse with membranes that contain endoplasmic reticulum (ER) resident proteins, including calreticulin and calnexin; trafficking with a compartment that contains the autophagosomal marker monodansylcadaverin; Brucella spp. replicate in an ER-like compartment; and then presumably lyse the host cell to disseminate and metastasize [25]. Macedo et al. found that susceptibility of MyD88 knockout (KO) mice to $B$. abortus infection is due to impaired dendritic cell (DC) maturation and lack of IL-12 production. Since DC maturation is a critical link between innate and adaptive immunity, MyD88-dependent signaling appears to be required for the development of IFN-gamma-producing T cells and efficient control of Brucella infection, providing insights into the orchestration of innate and adaptive immunity for control of B. abortus infection [26]. Toll-like receptors (TLRs), including TLR2, TLR4 and especially TLR9, interact with Brucella spp. and bacterial components and initiate mononuclear phagocyte responses that influence both innate and adaptive immunity. MyD88-dependent and Toll-IL-1 receptor (TIR)-domain-containing adapterinducing interferon-beta (TRIF)-independent signaling pathways are involved in Brucella activation of innate immune cells through TLRs [27].

Once inside the phagosome, the internalized Brucella surviving the initial oxidative burst and the nutrient/oxygen deprived environment subvert the host cellular machinery through the interaction of the pathogen's Type IV secretion system (virB) with the host's endoplasmic reticulum [24]. More recently, from the pathogen side of the interaction, Sengupta et al. have shown that Brucella spp. genomes encode a protein, named $\mathrm{TcpB}$, bearing homology with mammalian Toll/IL-1 receptor domains and whose expression causes degradation of the phosphorylated signal competent form of the adapter MyD88-adapter-like (MAL). Interestingly, the presence of TcpB leads to enhanced polyubiquitination of MAL and may be responsible for its accelerated degradation. The authors conclude that TcpB represents a unique pathogen-derived molecule that suppresses host innate-immune responses by specifically targeting an individual adapter molecule in the TLR signaling pathway for degradation [4]. The outcome of this host-pathogen interaction is prevention of lysosomal fusion, neutralization of phagosomal $\mathrm{pH}$, utilization of nitrate ions for anaerobic respiration, and regulated multiplication of the bacteria within the phagosome. The accumulated bacteria eventually escape the phagosome and are disseminated to other host cells. In female ruminant hosts, $B$. abortus interacts with the placental trophoblasts associated with the tissue tropism activity of erythritol produced by these cells. Within these cells, brucellae enter their acute replicative stage, with placental disruption resulting in fetal loss or the birth of weak, infected offspring [28]. The pathologic lesions of domestic and laboratory animals have been reviewed previously [9]. In humans, Brucella spread to the lymph nodes, spleen, liver and bone marrow with associated pathological manifestations of untreated infection often being meningitis, endocarditis, spondylitis, and arthritis [3]. 
The clinical presentation of human brucellosis can include fever, malaise, arthralgias, hepatomegaly, splenomegaly, lymphadenopathy, peripheral arthritis, sacroiliitis, epididymoorchitis, vomiting and diarrhea [29].

\section{MECHANISMS OF NATURAL DISEASE RESISTANCE TO BRUCELLOSIS}

\section{Historical and Biological Perspectives}

Natural (innate) resistance to bacterial diseases was observed over one hundred thirty years ago as familial tendencies in resistance or susceptibility to diphtheria in humans [30], but the genetic implications of this observation were not appreciated at that time, because it would be another twenty years until the rediscovery of Gregor Mendel's studies. In domestic livestock, it had been long observed that disease manifestations rarely occur in all members of the population exposed to bacterial pathogens, e.g. early studies of resistance to Salmonella pullorum in poultry [31] and B. suis in swine confirmed a major role for genetic control [32-34]. These early observations were largely ignored, because antibiotics were discovered in the late 1920 's, vaccines were developed for several animal diseases, and lastly, the genetics of natural disease resistance seemed unduly complicated, and there was concern that planned breeding programs to increase natural resistance would be too slow to have an impact and would compromise productivity. The animal genome always influences and sometimes determines susceptibility to bacterial diseases; however, due to the huge variety of pathogens and the multitude of complex host defense mechanisms involved, rarely does a simple understanding of resistance emerge. Although some of the observed variation in natural resistance is related to environmental factors, a significant component of variation in natural disease resistance is heritable and, therefore, stably passed from parent to offspring. The ability of a naive host to resist primary infection by a pathogen is known as natural disease resistance. F. B. Hutt specifically defined natural disease resistance as "the inherent capacity of an animal to resist disease when exposed to pathogens, without prior exposure or immunization, of which the major component is heritable and, therefore, stably passed from parent to offspring" [35, 36]. Given this series of complex host-pathogen interactions, it is obvious that control of natural bacterial infection and resulting disease would rarely be controlled by a single gene, although expression of an allele at one locus can significantly affect disease pathogenesis in individuals while at the herd and population levels, many genes would be operational in controlling the spectrum of disease expression. Obviously, selection for innate host resistance [37] should be considered as additive to one of several strategies, e.g. adaptive immunity through vaccination, hygiene and regulatory control measures, for integrated control of brucellosis. In attempt to balance host innate resistance and strategically poised Brucella escape mechanisms, we must also consider that once the selected innate resistance gene(s) spreads into the host population, Brucella variants expressing epitopes or other survival mechanisms that "innate resistant" animals fail to recognize or clear may be a consequence, as each strives for survival [38].

\section{Overview of Innate Resistance Mechanisms}

Natural disease resistance involves primarily the nonspecific immune response in vertebrates. Nonspecific immunity includes pattern-recognition receptors (PRRs) that bind to ancient and conserved bacterial PAMPs as well as single-stranded viral ribonucleic acid (ssRNA) [39], Tolllike receptors (TLRs) [40], nucleotide-binding oligomerization domain proteins (NODs) [41], lipopolysaccharide binding protein (LBP) [42], and mannose-binding protein (MBP) [43] are eukaryotic receptors that all recognize PAMPs. Binding of these PRRs with their target PAMPs results in opsonization, endocytosis or phagocytosis of the bacteria or virus, and initiates the transcription of nonspecific immune response genes. The products of these genes include antimicrobial peptides [44] and inflammatory cytokines [45]. This innate immune response, which occurs immediately after infection, serves to sequester the pathogen, limits its replication, and in some instances, clears the pathogen altogether. When the innate immune response is bypassed, evaded or overwhelmed, the adaptive immune response becomes paramount to host survival.

Phylogenetically the non-specific innate immune response predates the specific adaptive immune response. Innate immunity is found in some form or another in all multicellular organisms, while the adaptive immune response, appearing around 400 million years ago, is only found in vertebrates [46]. It was earlier thought that because the innate response was more primitive than the adaptive immune response, the innate response was also less advanced, and therefore not as important as the adaptive response. The innate immune response was thought to function merely as a stopgap measure during primary infection until the adaptive immune response could be activated. Additionally, because the innate immune response does not lead to immunological memory, it was considered less critical to overall immunity in higher organisms than was the adaptive immune response. However, there has been a paradigm shift in the scientific community with regards to the importance of the innate immune response [47], and the prevailing thought now is that innate immunity not only functions as the first line of defense against invading pathogens, but it also plays an instructive role in coordinating an effective adaptive immune response for short and long-term defense [48]. Thus, natural disease resistance, the nonspecific and specific immune responses both play significant roles in the outcome of Brucella infection.

\section{Innate Immunity Interactions with Adaptive Immunity}

In addition to the major role that BoLA plays in adaptive protective immunity, endogenous IL-12 produced during infection with $B$. abortus has been found to promote the production of IFN-gamma and the in vivo clearance of Brucella in mice by activating natural killer (NK) cells [49]. Human Vgamma9Vdelta2 T-cells play an important role in the early response to infection with intracellular pathogens, yet their number was dramatically increased in the peripheral blood of patients with acute brucellosis and specifically activated by non-peptidic low molecular weight 
compound(s) from $B$. suis lysate or by soluble factors produced by B. suis-infected macrophages [50].

\section{Host Cell Membrane Receptors}

Rezazadeh et al. investigated the role of human Toll-like receptor-4 (TLR4) polymorphisms that have been reported to reduce immune responsiveness to microbial pathogens, especially Brucella spp. They investigated the polymorphism in TLR4 gene (Asp299Gly) in patients with brucellosis and healthy volunteers matched for sex, age and geographic area. The $896 \mathrm{G}$ allele was significantly much more prevalent in patients with brucellosis compared to healthy controls Also the frequency of the G allele of TLR4 gene was significantly higher in male patients with brucellosis compared to the same sex in control group. Multiple logistic regression analysis found that male patients heterozygous at allele $G$ gene had a significantly higher risk for brucellosis with an odds ratio of 2.89 , revealing an association between the genetic polymorphism in TLR4 gene and susceptibility to brucellosis [51]. Hashemi et al. evaluated Fc gamma RIIa polymorphisms in patients with brucellosis such that the frequency of the two alleles and three genotypes for $\mathrm{Fc}$ gamma RIIa were virtually the same for H131 and R131 alleles in healthy controls and brucellosis patients, respectively. They concluded that the Fc gamma RIIa polymorphism was not decisive for the acquisition of brucellosis [52].

The A/C polymorphism (Ser128Arg) of E-selectin has been described to alter ligand binding function. Rafiei et al. found that the frequency of the Arg/Arg genotype of the Ser128Arg polymorphism was significantly increased in human brucellosis patients compared with healthy controls. After stratification of the patients according to disease duration, the association between the Arg allele and brucellosis was investigated disclosing that only in a subgroup of the patients with disease onset less than 38 weeks was the odds ratio significant, suggesting that the Arg/Arg genotype of the E-selectin gene polymorphism in codon 128 may be a genetic factor influencing susceptibility to Brucella infection [53]. Haidari et al. evaluated the singlenucleotide polymorphism C-159T in the promoter region of the CD14 gene that has been implicated in susceptibility to infectious diseases, and determined the CD14 genotype in human patients with brucellosis and healthy volunteers from the same rural area. The prevalence of genotype TT was significantly higher in the patients with brucellosis while the healthy controls had a higher prevalence of genotype CC. Furthermore, patients who were homozygous for allele $\mathrm{T}$ of promoter of CD14 gene had a significantly higher risk for developing brucellosis with odds ratio of 3.03 , thus their data provided evidence suggestive of an association of the CD14 gene polymorphism with susceptibility to brucellosis [54]. Since the host cellular prion protein $(\operatorname{PrP}(C))$ may function as a cell surface receptor and/or portal protein for B. abortus in mice [19], Seabury et al. evaluated the nucleotide and amino acid variation within exon 3 of the prion protein gene (PRNP) in US bison populations, identifying a nonsynonymous single nucleotide polymorphism (T50C), resulting in the predicted amino acid replacement M17T (Met --> Thr) while no variation (T50; Met) has yet been found in domestic cattle [55]. Interestingly, $80 \%$ of bison possessing the $\mathrm{C} / \mathrm{C}$ genotype were Brucella spp. seropositive and suggesting a potential association between nucleotide variation within PRNP exon 3 and the presence of Brucella spp. antibodies in bison, potentially implicating $\operatorname{Pr} P(C)$ in the natural resistance of bison to brucellosis infection [55].

\section{Cytokines}

Rafiei et al. investigated the association between transforming growth factor (TGF)-betal polymorphisms at codons 10 and 25 and brucellosis in human brucellosis patients and healthy volunteers. The frequencies of TGFbeta1 codons $10 \mathrm{C}$ and $25 \mathrm{G}$ were significantly higher among brucellosis patients than among healthy controls, as was that of TGF-betal codon $10 \mathrm{C} / \mathrm{C}$, suggesting that genetic polymorphism in codons 10 and 25 of the TGF-betal gene may contribute to the susceptibility of human brucellosis [56]. Since the interleukin-4 (IL-4) gene has a C-T substitution at position 590 which is associated with increased production of IL-4, Rezazadeh et al. investigated the potential association of this polymorphism with susceptibility to human brucellosis. The prevalence of the T allele of IL-4 polymorphism was significantly higher in the group with brucellosis than in controls, and furthermore patients with brucellosis had a higher frequency of intermediate producer genotype (CT) while low producer genotype (CC) was higher in the control group. Multiple logistic regression analysis demonstrated that patients who were heterozygous (CT) for interleukin-4 promoter polymorphism had a significantly higher risk for brucellosis with an odds ratio of 4.2, demonstrating an association between IL-4 590 promoter polymorphism and contracting human brucellosis [51].

\section{Macrophage Function}

Host resistance to $B$. abortus was found to be significantly related to macrophage function and immune mechanisms in cattle naturally resistant or susceptible to brucellosis $[57,58]$. Studies to determine the genetic basis for resistance to $B$. abortus in cattle found that natural resistance to brucellosis in cattle is a complex phenotypic trait determined by two or more interacting genes resulting in complex genetic types [59-61]. Twenty year classical breeding studies involving over 300 progeny of unvaccinated and unexposed sexually mature bulls and unvaccinated and unexposed 180 day pregnant outbred heifers phenotyped resistant or susceptible by standardized in vivo intraconjunctival challenge with $1 \times 10^{7} \mathrm{cfu}$ of $B$. abortus Strain 2308 established that the population frequency of natural resistance to Brucella could be significantly increased with one round of selective breeding [59, 61, 62]. In compliance with and approval by local, national and international health and animal protection and welfare requirements, the standard challenge is administered by bilaterally placing $50 \mu \mathrm{l}$ containing $5 \times 10^{6}$ cfu of B. abortus Strain 2308 in the conjunctiva sac such that the inoculum drains from each eye via the nasolacrimal duct into the nasal cavity, thus simulating a natural per os and/or aerosol exposure as would be expected to occur when ruminants are exposed by a Brucella induced abortion in a natural herd setting. Fortunately, classical genetic studies can now be greatly accelerated by embryo transfer, cloning technologies and now by deep sequencing methodologies. In these experiments, the frequency of natural resistance to 
standardized experimental $B$. abortus challenge was increased from $20 \%$ to almost $59 \%$ in one generation when mating a resistant bull to resistant heifers.

\section{Solute Carrier 11A1 (SLC11A1) (formerly Natural Disease Resistance-Macrophage Protein 1 (NRAMP1) Candidate Gene}

The solute carrier gene superfamily (SLC) encodes a group of integral membrane proteins that includes passive transporters, ion coupled transporters, and exchangers that traffic crucial compounds into and out of cells and organelles [63]. Currently the human SLC superfamily consists of 43 gene families encoding 298 individual transporter proteins [64]. A number of human disease syndromes are linked to SLC transporter gene defects, including hypertension [65], deafness [66], and hemochromatosis [67], and these integral membrane transporters proteins are prime candidates to be exploited as drug delivery systems or drug targets in the treatment of disease.

The SLC11 family consists of two member genes that encode proton coupled metal ion transporters, SLC11A1 and SLC11A2 (formerly known as DMT1). Originally characterized in mice, SLC11A1 mapped to the mouse $B c g / L s h / I t y$ locus, a region conferring resistance to infection by the live attenuated Bacillus Calmette-Guerin (BCG) strain of Mycobacterium bovis, Leishmania donovani, and Salmonella enterica serovar Typhimurium in the murine host [68]. SLC11A1 is a highly conserved gene, with orthologs found in both prokaryotes and eukaryotes, while homologs have been identified in mycobacteria [69], yeast [70], Drosophila [71], chickens [72], swine [73], dogs [74], horse [75], deer [76], cattle [77], and humans [78]. SLC11A1 expression occurs mainly in macrophages and is interferongamma-inducible. SLC11A1 expression in macrophages is also modulated by exposure to bacterial lipopolysaccharide (LPS) [79].

The SLC11A1 gene product is a polytopic integral membrane protein made up of 10-12 transmembrane domains. It is a 548 amino acid protein containing three putative phosphorylation sites, two SH3 binding motifs, and a single exofacial glycosylation site [80]. Bovine SLC11A1 also contains a highly conserved binding-protein-dependent transport system inner membrane component signature that alludes to the putative divalent cation transporter function of SLC11A1. Localization studies demonstrate that the protein product is recruited to the late endocytic compartment in a Lamp1 (lysosomal-associated membrane protein-1)-positive compartment and remains associated with this compartment as it matures to a phagolysosome [81], supporting the theory that SLC11A1 restricts replication of infecting pathogens by altering the intracellular environment. Research into the function of SLC11A1 suggests that the protein may modulate phagosomal $\mathrm{pH}$ [82], as well as alter divalent cation concentrations, including iron, within the phagosome $[83,84]$.

The disease resistance association of SLC11A1 inferred by the mapping the gene to the $B c g / L s h / I t y$ locus was confirmed in experiments in which innate resistance to intracellular pathogens was abrogated in SLC11A1 knockout mice [85]. In addition, the phosphoglycoprotein gene product of SLC11A1 was determined to be absent in macrophages from $B c g$ susceptible mouse strains, with antiSLC11A1 antiserum failing to detect mature SLC11A1 protein in macrophages isolated from $\mathrm{C} 57 \mathrm{BL} 6 / \mathrm{J}$ and $\mathrm{BALB} / \mathrm{c} B c g^{s}$ mice [86]. A single, non-conserved glycine-toaspartic acid amino acid substitution at position 169 results in a nonfunctional gene product and susceptibility of murine macrophages to $B C G$. In contrast to macrophages from $S L C 11 A 1^{\text {G169 }}$ mice, in which a $100-\mathrm{kDa}$ mature SLC11A1 protein is detected, SLC11A1 specific antiserum fails to detect an immunoreactive protein in macrophage lysates from susceptible mouse strains bearing the SLC11A1 DI69 allele [86]. This disrupting amino acid substitution is not found in SLC11A1 proteins sequenced from other species, including deer, bison, cattle, dogs, and humans. However, DNA sequence analysis and disease association studies in humans identified a functional microsatellite polymorphism in the human SLC11A1. In the human SLC11A1 gene, polymorphisms in a GT dinucleotide microsatellite in the 5, promoter of the gene correlate with both autoimmunity and infectious disease susceptibility [87]. A similar microsatellite length polymorphism was found in the 3' untranslated region of the bovine SLC11Algene [80]. Single Stranded Conformation Analysis (SSCA) indicated that length polymorphisms within this region of the gene were associated with disease resistance to $B$. abortus in cattle. Standardized in vivo wild type B. abortus challenge studies in unvaccinated, unexposed 180 day pregnant first calf heifers revealed that microsatellite lengths of 13 GT dinucleotide repeats were found in cattle resistant to challenge by $B$. abortus, while microsatellite lengths of 14 , 15 , and 16 GT dinucleotide repeats were found in cattle susceptible to B. abortus challenge [80]. As expected for a multigenic trait, a minority cattle with $\leq 13$ GT were susceptible, and a minority $\geq 14$ GT cattle were resistant, i.e. the association of natural resistance was not perfect for the GT polymorphisms of SLC11A1 in these studies [80]. Analysis of immune correlates related to natural resistance to Brucella revealed a differential response in macrophage activation between resistant and susceptible cattle, along with the associated segregation of specific alleles of bovine SLC11A1, a gene involved in macrophage activation [60]. In vitro assays demonstrated that macrophages from cattle with the resistant phenotype had a greater ability to kill $B$. abortus than did those from animals with the susceptible phenotype [88]. When an expression vector carrying the bovine SLC11A1 resistant allele (GT13) was transfected into a susceptible mouse macrophage cell line, the phenotype switched from susceptible to resistant in an in vitro macrophage-killing assay with $B$. abortus challenge [89]. Alternately, when the expression vector carrying a susceptible allele from bovine SLC11A1 (GT16) was transfected, there was no change in the susceptible phenotype. The same study found that a significantly higher level of SLC11A1 mRNA present in transfected cells carrying the resistant allele than in the transfected cells carrying the susceptible allele.

Horin [90] confirmed that the nucleotide sequence polymorphism due to a variation in the number of GT dinucleotide repeats is found in the 3' untranslated region (nucleotide positions 1781-1804) of the SLC11A1 gene, and identified different sequences with variable numbers of GT repeats, particularly $\mathrm{GT}_{10}$. The variation in the number of GT 
repeats was related to a variation in the number of 5, adjacent Gs. Deeper sequencing revealing nearly the complete structure of the bovine NRAMP 1 gene identified a novel polymorphism within intron $\mathrm{X}$ consisting of insertion of three guanine nucleotides at positions 37, 40 and 98 relative to the intron $\mathrm{X}$ start point, and scans of several cattle breeds suggested that the two intron $\mathrm{X}$ alleles identified are stable and widespread in the Bos taurus population [91]. Paixao et al. compared the frequency of SLC11A1 3'UTR polymorphisms between Holsteins (Bos taurus taurus) and Zebu (Bos taurus indicus), including the Nelore, Guzera, and Gir breeds. A marked difference in the frequency of alleles was detected between the Zebu and Holstein cattle that had only the GT13 genotype. The Nelore breed had the most heterogeneous genotype with four allelic combinations, namely, homozygous GT13, homozygous GT14, heterozygous GT13/GT14, and heterozygous GT13/GT15. The authors proposed that the allelic frequencies in different breeds of cattle may be useful in the future for planning breeding strategies for selection of naturally resistant cattle [92]. In an attempt to identify additional polymorphisms, Martinez et al. further characterized the SLC11A1 gene in different breeds of Colombian Creole Bos taurus and Bos indicus, identifying six new variants among a total of 11 single nucleotide mutations, of which five occurred in the coding sequence (three are missense mutations), one in the promoter region and five in introns [93, 94].

\section{PROPOSED ROLE OF SLC11A1 DINUCLEOTIDE REPEAT INSTABILITY ASSOCIATED WITH BRUCELLOSIS RESISTANCE}

In humans, instability in DNA dinucleotide and trinucleotide repeats is associated with several neurodegenerative and tumorigenic diseases. Genome wide instability of dinucleotide repeats is observed in tumors isolated from patients suffering from Hodgkin's disease and hereditary nonpolyposis colon cancer $[95,96]$. The archetypal triplet repeat disease, Huntington's disease, is categorized as a translated polyglutamine (polyQ) repeat disease, with its functionally associated unstable trinucleotide repeat (CAG), located in the first exon of the huntington $(\mathrm{Htt})$ gene, laying down an expanded tract of glutamines within the expressed sequence of the gene [95]. Repeat lengths of eight to 39 are found in normal individuals, while repeat lengths of 37 or more are found in affected individuals [97]. It is thought that the expressed polyQ tract results in a toxic gain of function of the mutant protein, such as formation of neuronal intranuclear inclusions, interference with cytoskeletal and vesicular transport, and impaired gene expression [98].

Microsatellite repeat diseases are not restricted solely to translated expansions within mutant protein. Repeat diseases with untranslated repeat expansions found in the 5' and 3' untranslated regions of the gene, as well as within the introns of the gene, have also been identified. Friedreich's ataxia (FRDA) is one such disease, containing an expanded GAA trinucleotide repeat in the first intron of the frataxin gene and inhibiting expression of the gene [99]. Although the mechanism of this altered gene regulation is unknown, one possibility is that changes in polymorphic microsatellite repeat can hinder binding of transcriptional factors through the localized formation of tightly packed heterochromatin or through disruption of synergistic protein-protein interactions and cooperative binding of the proteins to the DNA.

\section{PROTEIN-PROTEIN INTERACTIONS AND COOPERATIVE DNA BINDING OF REGULATORY FACTORS}

Nucleotide variations found in bovine SLC11A1 cDNA do not appear to be associated with natural disease resistance to $B$. abortus in cattle. Functionally associated nucleotide variants may be overlooked in under-represented alleles due to reduced expression levels of these alleles, and this is particularly important since it has been reported that individuals carrying the susceptible allele for SLC11A1 have reduced mRNA expression in both humans and cattle [80, 87]. Screening of the genomic complement of DNA for the sequence variation should provide a more accurate accounting of allelic variation in each individual since the DNA template would not be affected by allelic differences in expression. To date, the only bovine SLC11A1 polymorphisms proposed to be associated with $B$. abortus natural disease resistance in cattle is the dinucleotide microsatellite length polymorphism found in the 3 , untranslated region of the gene. While it is suggested from previous studies that variable microsatellite lengths affect macrophage bactericidal activity, the mechanism of this effect is not known. It is possible that changes in the 3'UTR microsatellite length may alter the angular orientation of two or more flanking transcription regulation sites specific for cooperatively binding transcription factors and reduce the efficiency of the synergistic binding of these proteins to the DNA molecule. In this case, polymorphisms in microsatellite length would alter transcription in a helical-phase dependent manner. Cooperative binding is a phenomenon whereby protein-protein interactions between two or more DNA binding proteins reduce the free energy of binding to DNA so that together proteins bind more tightly to DNA than each factor alone. This is a key mechanism for generating specificity within multicomponent nucleoprotein complexes [100]. DNA bending facilitates the protein-protein interactions between factors whose binding elements are separated by long distances across the DNA molecule [101]. Studies on cooperatively binding lambda repressor proteins have shown that changes in the angular orientation of the protein-binding site along the DNA alpha helix affects the strength of this protein-protein interaction [102]. Normally the two binding sites for lambda repressor have a center-tocenter distance of $25 \mathrm{bp}$ or 2.4 helical turns. Insertion of 10 or $11 \mathrm{bp}$ ( 1 helical increment) has no effect on cooperativity up to a distance of 80 bases or 8 turns of the a-helix. However, the insertion of non-helical increments abolishes co-operativity. This is due to the energetic penalty of twisting the DNA in order to place the proteins into a favorable angular orientation to interact cooperatively.

DISCREPANCIES IN THE ASSOCIATION OF THE POLYMORPHISMS OF THE CANDIDATE GENE, SCLC11A1, WITH PHENOTYPIC NATURAL DISEASE RESISTANCE TO BRUCELLOSIS

Evidence Supporting the Association of SLC11A1 with Natural Resistance against Brucellosis

Ganguly et al. evaluated the Murrah breed of buffalo (Bubalus bubalis) to identify four (GT13, GT14, GT15 and 
GT16) polymorphisms of the 3'UTR of SLC11A1 gene and evaluated the association of these polymorphisms with in vitro macrophage function. Non-vaccinated and serologically negative buffalo were divided into three genotypic groups: homozygous (GT)13 genotype; heterozygous [(GT)13/(GT)n, where $\mathrm{n} \neq 13]$; and non-(GT)13 $[(\mathrm{GT}) \mathrm{n} /(\mathrm{GT}) \mathrm{n}$, where $\mathrm{n} \neq 13]$, and macrophages were challenged with Brucella LPS to measure $\mathrm{H}_{2} \mathrm{O}_{2}$ and $\mathrm{NO}$ production. The homozygous [(GT)13/(GT)13] or heterozygous $[(\mathrm{GT}) 13 /(\mathrm{GT}) \mathrm{n}]$, where $\mathrm{n}=14,15$ or 16 ] (GT)13 allele, was significantly $(\mathrm{p}<0.01)$ associated with increased production of $\mathrm{H}_{2} \mathrm{O}_{2}$ and $\mathrm{NO}$, identifying the (GT)13 allelic variant to be significantly association with the improved macrophage function in buffalo [103]. Martinez et $a l$. further investigated the association between resistance to brucellosis infection and SLC11A1 SSCP genotype using a macrophage in vitro killing assay employing a virulent $B$. abortus strain. A significant association was found between the $B$. abortus macrophage in vitro killing assay phenotypes and the bovine SLC11A1 3' UTR genotypes, which suggested that the GT [12] A allele may be associated with resistance [94].

Borriello et al. tested water buffalo cows for the presence of anti-B. abortus antibodies and their corresponding SLC11A1 genotype and compared seropositive and seronegative groups with their respective SLC11A1 genotypes. The seropositive buffaloes had the SLC11A1A+ (SLC11A1AA or SLC11A1AB) genotype while the seronegatives had the SLC11A1A- (SLC11A1BB) genotype with an odds ratio of 4.37. Additionally, monocytes from SLC11A1BB buffalo had significantly higher levels of SLC11A1 mRNA than SLC11A1AA buffalo as well as a significantly enhanced ability to control the in vitro intracellular replication of several Brucella species, leading Borriello et al. to conclude that selection for the SLC11A1BB genotype may be a valuable tool for the control of brucellosis in water buffalo in endemic areas [104]. Capparelli et al. further evaluated the 3' untranslated region of the water buffalo $S L C 11 A 1$ gene to identify two alleles (SLC11A1A and SLC11A1B) in two independent populations of Brucella-seropositive and -seronegative control buffalo, and the SLC11A1AA genotype was associated with susceptibility. Moreover, macrophages from SLC11A1AA buffalo had a lower SLC11A1 mRNA level and a higher level of viable intracellular Brucella when compared with macrophages from SLC11A1BB buffalo. Also, monocytes and macrophages from SLC11A1AA subjects displayed a higher number of viable intracellular bacteria compared to SLC11A1BB animals, adding biological relevance to the association of genotypes with resistance or susceptibility [105]. Furthermore, Capparelli et al. tested water buffalo cows for the presence of anti-B. abortus antibodies and the SLC11A1 genotype. They detected four alleles (SLC11A1A, $-\mathrm{B},-\mathrm{C}$, and -D) in the 3' untranslated region of the SLC11A1 gene, and found that the SLC11A1BB genotype was represented among only the seronegatives, providing evidence that this genotype apparently confers resistance to B. abortus. The monocytes from the $S L C 11 A 1 \mathrm{BB}$ (resistant) buffaloes had a higher basal level of SLC11A1 mRNA and a lower number of viable intracellular bacteria than did the monocytes from SLC11A1AA (susceptible) genotyped buffaloes. Capparelli et al. proposed that the higher basal level of the antibacterial protein SLC11A1 probably provides the SLC11A1BB buffalos with the capability of controlling bacteria immediately after their entry inside the cell [106].

\section{Evidence Refuting the Association of SLC11A1 with Natural Resistance against Brucellosis}

Continuing their investigation of the bovine SLC11A1 gene, Paixao et al. evaluated the association between SLC11A1 3'UTR polymorphisms and resistance against bovine brucellosis in experimental and natural infections. In experimentally infected mixture of vaccinated and unvaccinated pregnant cows, abortion occurred in $42.1 \%$ of cows with a resistant genotype (SSCA(r) and in $43.1 \%$ of those with a susceptible genotype (SSCA(s). Additionally, the percentages of $B$. abortus positive cultures of the SSCA(r) genotype were 86 and $84 \%$ in serologically positive and negative cows from a farm with a very high prevalence of naturally-occurring bovine brucellosis. Hence, they found no association between the SLC11A1-resistant allele and the resistant phenotype in either experimental or naturally occurring brucellosis. Moreover, no differences were observed in the rates of intracellular survival of B. abortus within macrophages from cattle with susceptible or resistant genotypes. Thus, the investigators concluded that polymorphisms at the SLC11A1 3'UTR are not associated with resistance against $B$. abortus in cattle and that $S L C 11 A 1$ 3'UTR polymorphisms are not suitable markers of natural resistance against bovine brucellosis [107]. Bravo et al. examined polymorphisms of the SLC11A1 gene in human patients with brucellosis and healthy controls and found no significant differences in the alleles studied, concluding that variants of the SLC11A1 gene do not appear to affect susceptibility or protection in human brucellosis [108]. Kumar et al. evaluated samples from Indian zebu (Bos indicus) and crossbred (Bos indicus $x$ Bos taurus) cattle that were homozygous $(\mathrm{GT})(13) /(\mathrm{GT})(13)$. Cattle that were positive on three serological brucellosis tests with a history of abortion were grouped as "affected"; whereas the animals that were negative in all serological tests and completed third lactation without any history of abortion were grouped as "non-affected." The presence of (GT)(13) allele even in homozygous condition failed to provide resistance to brucellosis in a naturally infected herd [109]. In studies of bovine tuberculosis, even with high-level expression of SLC11A1 proteins in peripheral blood cells and granulomatous lesions heavily labeled epithelioid macrophages and Langhans cells, active tuberculosis was progressive [110]. In contrast to infections with Salmonella, Leishmania, and Mycobacterium, the expression of the SLC11A1 gene was found to be of limited importance for the natural resistance of mice to $B$. melitensis [111].

\section{The Challenge of the Association of the SLC11A1 with Resistance to Brucellosis}

It is not surprising that the prevailing hypothesis that polymorphisms at the bovine SLC11A1 3'UTR are associated with genetic resistance against $B$. abortus in cattle is being challenged, simply fulfilling the fundamental premise of the scientific process by which a hypothesis is only valid until it is proven wrong. As observed from the preceding series of publications, the role of the SLC11A1 
gene in resistance or susceptibility to ruminant brucellosis continues to be a hypothesis under serious investigation since the Adams and Templeton laboratories in the late 1980 's and early 1990 's demonstrated that resistance to $B$. abortus in cattle was a heritable trait and that polymorphisms of SLC11A1 3'UTR were associated with resistance, while fully recognizing that resistance to brucellosis was a complex phenotypic trait determined by two or more interacting genes. Clearly, data from other laboratories support (or refute) this hypothesis that polymorphisms at the bovine SLC11A1 3'UTR are (are not) associated with genetic resistance against $B$. abortus in cattle. The authors of other publications suggest that SLC11A1 3'UTR polymorphisms are not suitable markers of natural resistance against bovine brucellosis, while the results from other laboratories support the hypothesis.

There are a number of differences in designs, phenotyping protocols, performance standards and execution of the experiments from various laboratories that make it difficult to directly confront the discrepancies in the results and the subsequent interpretation of the data. The genetic background of the experimental animals is particularly relevant for several reasons: i) natural resistance/susceptibility to B. abortus is multigenic and all the genes encoding this phenotype have not been clearly defined; ii) even within a genetically-defined population of cattle, the correlation between the in vivo phenotype -as determined by experimental infection and the in vitro phenotype -as determined by in vitro killing assays- is not absolute; iii) there are likely breed-specific differences in frequencies of natural resistance/susceptibility to infectious diseases in cattle, e.g. 100\% frequency of bovine NRAMP1 $\mathrm{SSCA}^{\mathrm{r}}$ alleles in the Brazilian Holstein cattle reported by Paixao et al. [107]. Additionally, adoption of internationally standardized phenotyping of bovine brucellosis resistance or susceptibility by in vivo challenge with $1 \times 10^{7}$ cfu of wild type B. abortus Strain 2308 in unvaccinated, unexposed first calf heifers at $180 \pm 30$ days gestation without recovery of the pathogen from any tissues or secretions from the dam, placenta and fetus should constitute the resistance phenotype which would be highly desirable to evaluate the phenotypes in virtually any bovine population anywhere. Accordingly, the criteria applied by Paixao et al. [107] to determine the in vivo phenotype of the experimental cattle ("for the purpose of this study, susceptibility to brucellosis was characterized by the occurrence of abortion and by the intensity of inflammatory lesions in several organs") were certainly different if not less stringent than those used in original phenotyping experiments of Adams and Templeton [58, 61$62,112]$. Certainly, according to Hutt's original description of natural disease resistance, cattle should not be vaccinated for brucellosis or have had natural exposure to the Brucella prior to phenotyping by virulent challenge as occurred in the Paixao et al. [107] experiments in which 29 of the 42 heifers were previously vaccinated with either S19 or RB51 ("The inclusion of vaccinated cattle in this study was due to the fact that a significant percentage of the female bovine population are vaccinated, and therefore a marker for natural resistance should be suitable for both vaccinated and non vaccinated cattle"), or regarding the unconfirmed vaccination status of the cattle herd used in the natural Brucella infection study, in which there were "no records of vaccination against brucellosis were available for this herd." The obvious concern with cattle, whether vaccinated or not, naturally exposed to B. abortus is that it is not possible to confirm the time of exposure relative to gestation, the dosage of exposure and the virulence of the wild type organism any or all of which seriously compromise the phenotype classification. From the data cited above of the phenotyping experiments reviewed here and the subsequent discussion of the data, it is obvious that the procedures for in vivo phenotyping resistance against Brucella in ruminants are complex and should be conducted using internationally accepted standardized guidelines for challenge conditions and phenotyping assessment protocols to improve the efficiency of identifying the subtleties of the multigenic traits of natural resistance mechanisms against brucellosis.

\section{SUMMARY}

Natural resistance against brucellosis was reviewed from historical and biological perspectives with regard to animals, humans and Brucella spp. Unfortunately, brucellosis continues to be a serious worldwide bacterial zoonosis of major significance to animal and human populations. Host genetic, innate and adaptive immune factors significantly influence the outcome of brucellosis as does the enabling strategies of intracellular Brucella to evade host factors resulting in a delicate co-evolutionary balance for long term survival for both host and pathogen. Given this series of complex host-pathogen interactions, it seems clear that control of brucellosis and resulting disease would rarely be controlled by a single gene, although expression of an allele at one locus can significantly affect disease pathogenesis in individuals while at the herd and population levels, many genes are likely to be operational in controlling the spectrum of disease expression. Natural (innate) resistance mechanisms includes the complex of host cell surface receptors for Brucella pathogen-associated molecular patterns, Toll-IL-1 receptor mediated pathways, factors mediating effective macrophage and dendritic cell maturation and activation, carbohydrate binding proteins, antimicrobial peptides, and inflammatory cytokines orchestrated and regulated by the host genome. Heritability of natural resistance has long been recognized as a complex multigenic trait, however new tools for understanding the genetic basis for innate resistance are now providing a deeper knowledge to identify genes and polymorphisms associated with resistance or susceptibility. For example, polymorphisms in TGFbeta 1, E-selectin, TLR4, CD14 genes and the IL-4 promoter were found to be associated with a higher risk for contracting human brucellosis, while the Fc gamma RIIa was not decisive for susceptibility to brucellosis. Polymorphisms of the 3'UTR of the candidate gene, SLC11A1, have been investigated extensively in numerous host species yielding variable degrees of association with natural (innate) resistance to brucellosis. Publications supporting or refuting the hypothesis of associating SLC11A1 3'UTR polymorphisms with resistance to brucellosis in cattle and water buffalo were reviewed clearly indicating that further investigation will be required using standardized in vivo phenotyping guidelines to resolve the controversy and potential use of the SLC11A1 3'UTR polymorphisms as a basis for selecting ruminants naturally 
resistant to B. abortus. Given the rate of development of new genetic tools coupled with rigorously controlled phenotyping protocols, it is anticipated that applying genetic selection as additional approach to controlling infectious diseases, such as brucellosis, in domestic animals will become increasingly feasible in the future.

\section{ACKNOWLEDGEMENTS}

The authors posthumously dedicate this review to Professor Joe W. Templeton long term colleague and friend who collaborated with us for 30 thirty years pursuing genetic disease resistance in domestic animals. The authors acknowledge the support of Texas AgriLife Research Projects H-6194 and H-8409, United States Department of Agriculture - Cooperative State Research Service grants 9037241-5583 and 93-37204-9491, and Formula Animal Health Grant USDA-FATEX 0-8050, the Texas Advanced Technology Research Program, and the USDAOICD/Egyptian National Agriculture Research Project - 58319R-3-005.

$$
\begin{aligned}
& \text { ABBREVIATIONS } \\
& \mathrm{BCV}=\text { Brucella-Containing Vacuole } \\
& \text { CR3 = Complement Receptor } 3(\mathrm{CD} 11 \mathrm{~b} / \mathrm{CD} 18) \\
& \text { DC }=\text { Dendritic Cell } \\
& \text { Fc } \quad=\text { Fragment, crystallizable of Immunoglobulins } \\
& \text { LAMP1 = Lysosomal-Associated Membrane Protein } 1 \\
& \text { LBP = Lipopolysaccharide Binding Protein } \\
& \text { LFA-1 = Leukocyte Function-Associated Antigen } 1 \\
& \text { (CD18/CD11a) } \\
& \text { MAL = MyD88-Adapter-Like } \\
& \text { MBP = Mannose Binding Protein } \\
& \text { MPR = Mannose 6-Phosphate Receptors } \\
& \text { NK } \quad=\text { Natural Killer Cell } \\
& \text { NOD = Nucleotide-Binding Oligomerization Domain } \\
& \text { NRAMP1 = Natural Resistance-Associated Macrophage } \\
& \text { Protein } 1 \text { gene } \\
& \text { PAMP = Pathogen-Associated Molecular Patterns } \\
& \text { PRR = Pattern-Recognition Receptors } \\
& \text { SLC11A1 = Solute Carrier 11A1 gene } \\
& \text { TLR = Toll-like Receptor } \\
& \text { TRIF = Toll-IL-1 Receptor (TIR)-Domain-Containing } \\
& \text { Adapter-Inducing Interferon-Beta } \\
& \text { UTR = Untranslated Region }
\end{aligned}
$$

\section{REFERENCES}

[1] Bruce D. The Micrococcus of Malta Fever. Practicioner 1888; 40: 2341-9.

[2] Corbel MJ. Brucellosis: an overview. Emerg Infect Dis 1997; 3(2): 213-21.

[3] Ko J, Splitter GA. Molecular host-pathogen interaction in brucellosis: current understanding and future approaches to vaccine development for mice and humans. Clin Microbiol Rev 2003; 16(1): 65-78.
[4] Sengupta D, Koblansky A, Gaines J, et al. Subversion of innate immune responses by Brucella through the targeted degradation of the TLR signaling adapter, MAL. J Immunol 2010; 184(2): 956-64.

[5] Diacovich L, Gorvel JP. Bacterial manipulation of innate immunity to promote infection. Nat Rev Microbiol 2010; 8(2): 117-28.

[6] Templeton JW, Smith R, Adams LG. Natural disease resistance in domestic animals. J Am Vet Med Assoc 1988; 192(9): 1306-15.

[7] Ishii KJ, Koyama S, Nakagawa A, et al. Host innate immune receptors and beyond: making sense of microbial infections. Cell Host Microbe 2008; 3(6): 352-63.

[8] Horin P. Biological principles of heredity of and resistance to disease. Rev Sci Tech 1998; 17(1): 302-14.

[9] Adams LG. The pathology of brucellosis reflects the outcome of the battle between the host genome and the Brucella genome. Vet Microbiol 2002; 90(1-4): 553-61.

[10] Lengeling A, Pfeffer K, Balling R. The battle of two genomes: genetics of bacterial host/pathogen interactions in mice. Mamm Genome 2001; 12(4): 261-71.

[11] Taleski V, Zerva L, Kantardjiev T, et al. An overview of the epidemiology and epizootology of brucellosis in selected countries of Central and Southeast Europe. Vet Microbiol 2002; 90(1-4): 147-55.

[12] Corbel MJ. Recent advances in brucellosis. J Med Microbiol 1997; 46(2): 101-3

[13 Greenfield RA, Drevets DA, Machado LJ, et al. Bacterial pathogens as biological weapons and agents of bioterrorism. Am J Med Sci 2002; 323(6): 299-315.

[14] Anonymous. From the Centers for Disease Control and Prevention. Suspected brucellosis case prompts investigation of possible bioterrorism-related activity--New Hampshire and Massachusetts, 1999. JAMA 2000; 284(3): 300-2.

[15] Anonymous. United and Strengthening America by Providing Appropriate Tools Required to Intersept and Obstruct Terroriam Act of 2001, October 24, 2001.

[16] Finlay BB, Falkow S. Common themes in microbial pathogenicity. Microbiol Rev 1989; 53(2): 210-30.

[17] Campbell GA, Adams LG, Sowa BA. Mechanisms of binding of Brucella abortus to mononuclear phagocytes from cows naturally resistant or susceptible to brucellosis. Vet Immunol Immunopathol 1994; 41(3-4): 295-306.

[18] Kim S, Watarai M, Suzuki H, et al. Lipid raft microdomains mediate class A scavenger receptor-dependent infection of Brucella abortus. Microb Pathog 2004; 37(1): 11-9.

[19] Watarai M. Interaction between Brucella abortus and cellular prion protein in lipid raft microdomains. Microbes Infect 2004; 6(1): 93100.

[20] Watarai M, Makino S, Fujii Y, et al. Modulation of Brucellainduced macropinocytosis by lipid rafts mediates intracellular replication. Cell Microbiol 2002; 4(6): 341-55.

[21] Watarai M, Makino S, Michikawa M, et al. Macrophage plasma membrane cholesterol contributes to Brucella abortus infection of mice. Infect Immun 2002; 70(9): 4818-25.

[22] Capparelli R, Parlato M, Amoroso MG, et al. Mannose-binding lectin haplotypes influence Brucella abortus infection in the water buffalo (Bubalus bubalis). Immunogenetics 2008; 60(3-4): 157-65.

[23] Rittig MG, Alvarez-Martinez MT, Porte F, et al. Intracellular survival of Brucella spp. in human monocytes involves conventional uptake but special phagosomes. Infect Immun 2001; 69(6): 3995-4006.

[24] Celli J, Salcedo SP, Gorvel JP. Brucella coopts the small GTPase Sar1 for intracellular replication. Proc Natl Acad Sci USA 2005; 102(5): 1673-8.

[25] Qin QM, Pei J, Ancona V, et al. RNAi screen of endoplasmic reticulum-associated host factors reveals a role for IRE1alpha in supporting Brucella replication. PLoS Pathog 2008; 4(7): e1000110.

[26] Macedo GC, Magnani DM, Carvalho NB, et al. Central role of MyD88-dependent dendritic cell maturation and proinflammatory cytokine production to control Brucella abortus infection. J Immunol 2008; 180(2): 1080-7.

[27] Oliveira SC, de Oliveira FS, Macedo GC, et al. The role of innate immune receptors in the control of Brucella abortus infection: tolllike receptors and beyond. Microbes Infect 2008; 10(9): 1005-9.

[28] Cutler SJ, Whatmore AM, Commander NJ. Brucellosis--new aspects of an old disease. J Appl Microbiol 2005; 98(6): 1270-81. 
[29] Pappas G, Akritidis N, Bosilkovski M, et al. Brucellosis. N Engl J Med 2005; 352(22): 2325-36.

[30] Jacobi A. Treatise on diphtheria. New York: William Wood \& Co 1880.

[31] Roberts E, Card L. The inheritance of bacillary white diarrhea. Poult Sci 1926; 6: 18-23.

[32] Cameron HS, Hughes EH, Gregory PW. Genetic resistance to brucellosis in swine. Cornell Vet 1940; 1: 18-23.

[33] Cameron HS, Hughes EH, Gregory PW. Genetic resistance to brucellosis in swine. J Anim Sci 1942; 1: 106-10.

[34] Cameron HS, Hughes EH, Gregory PW. Inherited resistance to brucellosis in inbred Berkshire swine. Am J Vet Res 1943; 3: 3879.

[35] Hutt FB. Genetic resistance to disease in domestic animals. Ithaca, NY: Comstock Publishing Associates 1958.

[36] Hutt FB. Animal genetics. New York: Ronald Press 1962.

[37] Parham P. Innate immunity: The unsung heroes. Nature 2003; 423(6935): 20.

[38] Scalzo AA. Successful control of viruses by NK cells--a balance of opposing forces? Trends Microbiol 2002; 10(10): 470-4.

[39] Medzhitov R, Janeway CA, Jr. Innate immunity: impact on the adaptive immune response. Curr Opin Immunol 1997; 9(1): 4-9.

[40] Takeda K, Kaisho T, Akira S. Toll-like receptors. Annu Rev Immunol 2003; 21: 335-76.

[41] Chamaillard M, Girardin SE, Viala J, et al. Nods, Nalps and Naip: intracellular regulators of bacterial-induced inflammation. Cell Microbiol 2003; 5(9): 581-92.

[42] Wright SD, Ramos RA, Tobias PS, et al. CD14, a receptor for complexes of lipopolysaccharide (LPS) and LPS binding protein. Science 1990; 249(4975): 1431-3.

[43] Stahl PD, Ezekowitz RA. The mannose receptor is a pattern recognition receptor involved in host defense. Curr Opin Immunol 1998; 10(1): 50-5.

[44] Ganz T, Lehrer RI. Antimicrobial peptides of vertebrates. Curr Opin Immunol 1998; 10(1): 41-4.

[45] Barnes B, Lubyova B, Pitha PM. On the role of IRF in host defense. J Interferon Cytokine Res 2002; 22(1): 59-71.

[46] Thompson CB. New insights into V(D)J recombination and its role in the evolution of the immune system. Immunity 1995; 3(5): 5319 .

[47] Coutinho A, Poltorack A. Innate immunity: from lymphocyte mitogens to Toll-like receptors and back. Curr Opin Immunol 2003; 15(6): 599-602.

[48] Fearon DT, Locksley RM. The instructive role of innate immunity in the acquired immune response. Science 1996; 272(5258): 50-3.

[49] Zhan Y, Cheers C. Endogenous interleukin-12 is involved in resistance to Brucella abortus infection. Infect Immun 1995; 63(4): 1387-90.

[50] Dornand J, Gross A, Lafont V, et al. The innate immune response against Brucella in humans. Vet Microbiol 2002; 90(1-4): 383-94.

[51] Rezazadeh M, Hajilooi M, Haidari M, et al. Association of susceptibility to brucellosis and interleukin-4 promoter polymorphism. Scand J Infect Dis 2006; 38(11-12): 1045-9.

[52] Hashemi SH, Hajilooi M, Mamani M, et al. Fc gamma receptor IIa polymorphism in patients with brucellosis. Jpn J Infect Dis 2007; 60(4): 196-7.

[53] Rafiei A, Hajilooi M, Vahedi M, et al. The Ser128Arg polymorphism for E-selectin gene and brucellosis. Infect Genet Evol 2007; 7(4): 494-8.

[54] Haidari M, Hajilooi M, Rezazadeh M, et al. Polymorphism in the promoter region of the CD14 gene and susceptibility to Brucellosis. Immunol Invest 2006; 35(2): 239-45.

[55] Seabury CM, Halbert ND, Gogan PJ, et al. Bison PRNP genotyping and potential association with Brucella spp. seroprevalence. Anim Genet 2005; 36(2): 104-10.

[56] Rafiei A, Hajilooi M, Shakib RJ, et al. Transforming growth factorbeta1 polymorphisms in patients with brucellosis: an association between codon 10 and 25 polymorphisms and brucellosis. Clin Microbiol Infect 2007; 13(1): 97-100.

[57] Price RE, Templeton JW, Smith R, et al. Ability of mononuclear phagocytes from cattle naturally resistant or susceptible to brucellosis to control in vitro intracellular survival of Brucella abortus. Infect Immun 1990; 58(4): 879-86.

[58] Harmon BG, Templeton JW, Crawford RP, et al. Macrophage function and immune response of Brucella abortus naturally resistant and susceptible cattle. In: Skamene E, Ed. New York: Alan R. Liss, Inc. 1985.

[59] Templeton JW, Price RE, Estes DM, et al. Genetics of natural resistance to Brucella abortus in cattle. Immunobiology 1989; 4: 143-7.

[60] Adams LG, Templeton JW. Genetic resistance to bacterial diseases of animals. Rev Sci Tech 1998; 17: 200-19.

[61] Templeton JW, Estes DM, Price RE, Smith R, III, Adams LG, Eds. Immunogenetics of natural resistance to bovine brucellosis. 4th World Congress on Genetics Applied to Livestock Production; Dunedin, NZ 1990.

[62] Templeton JW, Adams LG. Natural resistance to bovine brucellosis. In: Adams LG, Ed. Advances in Brucellosis research: An International Symposium. College Station, TX: Texas A\&M University Press 1990; pp. 144-50.

[63] Forbes JR, Gros P. Iron, manganese, and cobalt transport by Nramp1 (Slc11a1) and Nramp2 (Slc11a2) expressed at the plasma membrane. Blood 2003; 102(5): 1884-92.

[64] Hediger MA, Romero MF, Peng JB, et al. The ABCs of solute carriers: physiological, pathological and therapeutic implications of human membrane transport proteinsIntroduction. Pflugers Arch 2004; 447(5): 465-8

[65] Hebert SC, Mount DB, Gamba G. Molecular physiology of cationcoupled Cl- cotransport: the SLC12 family. Pflugers Arch 2004; 447(5): 580-93.

[66] Mount DB, Romero MF. The SLC26 gene family of multifunctional anion exchangers. Pflugers Arch 2004; 447(5): $710-21$

[67] McKie AT, Barlow DJ. The SLC40 basolateral iron transporter family (IREG1/ferroportin/MTP1). Pflugers Arch 2004; 447(5): 801-6.

[68] Vidal S, Gros P, Skamene E. Natural resistance to infection with intracellular parasites: molecular genetics identifies Nrampl as the Bcg/Ity/Lsh locus. J Leukoc Biol 1995; 58(4): 382-90.

[69] Agranoff D, Monahan IM, Mangan JA, et al. Mycobacterium tuberculosis expresses a novel pH-dependent divalent cation transporter belonging to the Nramp family. J Exp Med 1999; 190(5): 717-24.

[70] Liu XF, Culotta VC. Post-translation control of Nramp metal transport in yeast. Role of metal ions and the BSD2 gene. J Biol Chem 1999; 274(8): 4863-8

[71] Rodrigues V, Cheah PY, Ray K, et al. malvolio, the Drosophila homologue of mouse NRAMP-1 (Bcg), is expressed in macrophages and in the nervous system and is required for normal taste behaviour. EMBO J 1995; 14(13): 3007-20.

[72] Hu J, Bumstead N, Burke D, et al. Genetic and physical mapping of the natural resistance-associated macrophage protein 1 (NRAMP1) in chicken. Mamm Genome 1995; 6(11): 809-15.

[73] Tuggle CK, Schmitz CB, Gingerich-Feil D. Rapid communication: cloning of a pig full-length natural resistance associated macrophage protein (NRAMP1) cDNA. J Anim Sci 1997; 75(1): 277.

[74] Altet L, Francino O, Solano-Gallego L, et al. Mapping and sequencing of the canine NRAMPI gene and identification of mutations in leishmaniasis-susceptible dogs. Infect Immun 2002; 70(6): 2763-71.

[75] Horin P, Matiasovic J. Two polymorphic markers for the horse SLC11A1 (NRAMP1) gene. Anim Genet 2000; 31(2): 152.

[76] Matthews GD, Crawford AM. Cloning, sequencing and linkage mapping of the NRAMPI gene of sheep and deer. Anim Genet 1998; 29(1): 1-6.

[77] Feng J. Isolation and characterization of bovine Nrampl and bison Nrampl-A major candidate gene for natural resistance to Brucellosis College Station. TX: Texas A\&M University 1996.

[78] Kishi F. Isolation and characterization of human Nramp cDNA. Biochem Biophys Res Commun 1994; 204(3): 1074-80.

[79] Atkinson PG, Blackwell JM, Barton CH. Nramp1 locus encodes a $65 \mathrm{kDa}$ interferon-gamma-inducible protein in murine macrophages. Biochem J 1997; 325 (3): 779-86.

[80] Feng J, Li Y, Hashad M, et al. Bovine natural resistance associated macrophage protein 1 (Nramp1) gene. Genome Res 1996; 6(10): 956-64.

[81] Gruenheid S, Pinner E, Desjardins M, et al. Natural resistance to infection with intracellular pathogens: the Nramp1 protein is recruited to the membrane of the phagosome. J Exp Med 1997; 185(4): 717-30. 
[82] Hackam DJ, Rotstein OD, Zhang W, et al. Host resistance to intracellular infection: mutation of natural resistance-associated macrophage protein 1 (Nrampl) impairs phagosomal acidification. J Exp Med 1998; 188(2): 351-64.

[83] Barton CH, Biggs TE, Baker ST, et al. Nramp1: a link between intracellular iron transport and innate resistance to intracellular pathogens. J Leukoc Biol 1999; 66(5): 757-62.

[84] Wyllie S, Seu P, Goss JA. The natural resistance-associated macrophage protein 1 Slc1la1 (formerly Nramp1) and iron metabolism in macrophages. Microbes Infect 2002; 4(3): 351-9.

[85] Vidal S, Tremblay ML, Govoni G, et al. The Ity/Lsh/Bcg locus: natural resistance to infection with intracellular parasites is abrogated by disruption of the Nrampl gene. J Exp Med 1995; 182(3): 655-66.

[86] Vidal SM, Pinner E, Lepage $P$, et al. Natural resistance to intracellular infections: Nrampl encodes a membrane phosphoglycoprotein absent in macrophages from susceptible (Nramp1 D169) mouse strains. J Immunol 1996; 157(8): 3559-68.

[87] Searle S, Blackwell JM. Evidence for a functional repeat polymorphism in the promoter of the human NRAMP1 gene that correlates with autoimmune versus infectious disease susceptibility. J Med Genet 1999; 36(4): 295-9.

[88] Qureshi T, Templeton JW, Adams LG. Intracellular survival of Brucella abortus, Mycobacterium bovis BCG, Salmonella dublin, and Salmonella typhimurium in macrophages from cattle genetically resistant to Brucella abortus. Vet Immunol Immunopathol 1996; 50(1-2): 55-65.

[89] Barthel R, Feng J, Piedrahita JA, et al. Stable transfection of the bovine NRAMP1 gene into murine RAW264.7 cells: effect on Brucella abortus survival. Infect Immun 2001; 69(5): 3110-9.

[90] Horin P, Rychlik I, Templeton JW, et al. A complex pattern of microsatellite polymorphism within the bovine NRAMP1 gene. Eur J Immunogenet 1999; 26(4): 311-3.

[91] Coussens PM, Coussens MJ, Tooker BC, et al. Structure of the bovine natural resistance associated macrophage protein (NRAMPI) gene and identification of a novel polymorphism. DNA Seq 2004; 15(1): 15-25.

[92] Paixao TA, Ferreira C, Borges AM, et al. Frequency of bovine Nramp1 (Slcllal) alleles in Holstein and Zebu breeds. Vet Immunol Immunopathol 2006; 109(1-2): 37-42.

[93] Martinez R, Toro R, Montoya F. Evaluacion genetica para resistencia a brucelosis en ganado criollo colombiano BON. Arch Zootecnia 2005: 206-7: 333-40.

[94] Martinez R, Toro R, Montoya F, et al. Bovine SLC11A1 3' UTR SSCP genotype evaluated by a macrophage in vitro killing assay employing a Brucella abortus strain. J Anim Breed Genet 2008; 125(4): 271-9.

[95] Aaltonen LA, Peltomaki P, Leach FS, et al. Clues to the pathogenesis of familial colorectal cancer. Science 1993; 260(5109): 812-6.

[96] Mark Z, Toren A, Amariglio N, et al. Instability of dinucleotide repeats in Hodgkin's disease. Am J Hematol 1998; 57(2): 148-52.
[97] Rubinsztein DC, Leggo J, Coles R, et al. Phenotypic characterization of individuals with 30-40 CAG repeats in the Huntington disease (HD) gene reveals HD cases with 36 repeats and apparently normal elderly individuals with 36-39 repeats. Am J Hum Genet 1996; 59(1): 16-22.

[98] Everett CM, Wood NW. Trinucleotide repeats and neurodegenerative disease. Brain 2004; 127(11): 2385-405.

[99] Campuzano V, Montermini L, Molto MD, et al. Friedreich's ataxia: autosomal recessive disease caused by an intronic GAA triplet repeat expansion. Science 1996; 271(5254): 1423-7.

[100] Ozer J, Mitsouras K, Zerby D, et al. Transcription factor IIA derepresses TATA-binding protein (TBP)-associated factor inhibition of TBP-DNA binding. J Biol Chem 1998; 273(23): 14293-300.

[101] Crothers DM, Gartenberg MR, Shrader TE. DNA bending in protein-DNA complexes. Methods Enzymol 1991; 208: 118-46.

[102] Hochschild A, Ptashne M. Cooperative binding of lambda repressors to sites separated by integral turns of the DNA helix. Cell 1986; 44(5): 681-7.

[103] Ganguly I, Sharma A, Singh R, et al. Association of microsatellite (GT)n polymorphism at 3'UTR of NRAMPI with the macrophage function following challenge with Brucella LPS in buffalo (Bubalus bubalis). Vet Microbiol 2008; 129(1-2): 188-96.

[104] Borriello G, Capparelli R, Bianco M, et al. Genetic resistance to Brucella abortus in the water buffalo (Bubalus bubalis). Infect Immun 2006; 74(4): 2115-20.

[105] Capparelli R, Alfano F, Amoroso MG, et al. Protective effect of the Nramp1 BB genotype against Brucella abortus in the water buffalo (Bubalus bubalis). Infect Immun 2007; 75(2): 988-96.

[106] Capparelli R, Borriello G, Marabelli R, et al. The Nrampl AA genotype confers susceptibility to Brucella abortus in water buffalo. Mamm Genome 2007; 18(2): 137-43.

[107] Paixao TA, Poester FP, Neta CAV, et al. NRAMP1 3' untranslated region polymorphisms are not associated with natural resistance to Brucella abortus in cattle. Infect Immun 2007; 75(5): 2493-9.

[108] Bravo MJ, Colmenero Jde D, Martin J, et al. Variation in the NRAMP1 gene does not affect susceptibility or protection in human brucellosis. Microbes Infect 2006; 8(1): 154-6.

[109] Kumar N, Mitra A, Ganguly I, et al. Lack of association of brucellosis resistance with (GT)(13) microsatellite allele at 3'UTR of NRAMPl gene in Indian zebu (Bos indicus) and crossbred (Bos indicus x Bos taurus) cattle. Vet Microbiol 2005; 111(1-2): 139-43.

[110] Estrada-Chavez C, Pereira-Suarez AL, Meraz MA, et al. High-level expression of NRAMPI in peripheral blood cells and tuberculous granulomas from Mycobacterium bovis-infected bovines. Infect Immun 2001; 69(11): 7165-8.

[111] Guilloteau LA, Dornand J, Gross A, et al. Nramp1 is not a major determinant in the control of Brucella melitensis infection in mice. Infect Immun 2003; 71(2): 621-8.

[112] Adams LG, Barthel R, Pabello GJA, et al. Bovine natura resistance macrophage protein 1 (NRAMPl) gene. Arch Tierz 1999; 42: 42-56.

This is an open access article licensed under the terms of the Creative Commons Attribution Non-Commercial License (http://creativecommons.org/licenses/by-nc/ $3.0 /$ ) which permits unrestricted, non-commercial use, distribution and reproduction in any medium, provided the work is properly cited. 